\title{
Prognostic Value of Log Odds of Positive Lymph Nodes after Radical Surgery Followed by Adjuvant Treatment in High-Risk Cervical Cancer
}

\author{
Jeanny Kwon, $\mathrm{MD}^{1}$ \\ Keun-Yong Eom, MD, PhD² \\ In Ah Kim, MD, PhD² \\ Jae-Sung Kim, MD, PhD² \\ Young-Beom Kim, MD, PhD \\ Jae Hong No, MD, $\mathrm{PhD}^{3}$ \\ Kidong Kim, MD, PhD
}

\begin{abstract}
Purpose
The purpose of this study is to compare the prognostic efficacy of the number and location of positive lymph nodes (LN), LN ratio (LNR), and log odds of positive LNs (LODDs) in highrisk cervical cancer treated with radical surgery and adjuvant treatment.
\end{abstract}

\section{Materials and Methods}

Fifty high-risk patients who underwent radical hysterectomy and pelvic node dissection followed by adjuvant treatment were analyzed retrospectively. The patients had International Federation of Gynecology and Obstetrics (FIGO) stage IA2-IIB. Upper LN is defined as common iliac or higher LN, and LNR is the ratio of positive LNs to harvested LNs. LODDs is log odds between positive LNs and negative LNs. Radiotherapy was delivered to the whole pelvis with median $50.4 \mathrm{~Gy} / 28 \mathrm{Fx} \pm$ to the para-aortic regions. Platinum-based chemotherapy was used in most patients (93\%). The median follow-up duration was 80 months.

\section{Results}

The 5-year disease-free survival (DFS) rate was $76.1 \%$, and the overall survival (OS) rate was $86.4 \%$. Treatment failure occurred in 11 patients, and distant failure (DF) was the dominant pattern (90.9\%). In univariate analysis, significantly lower DFS was observed in patients with perineural invasion, $\geq 2$ LN metastases, LNR $\geq 10 \%$, upper LN metastasis, and $\geq-1.05$ LODDs. In multivariate analysis, $\geq-1.05$ LODDs was the only significant factor for DFS ( $p=0.011$ ). Of patients with LODDs $\geq-1.05,40.9 \%$ experienced DF. LODDs was the only significant prognostic factor for OS as well $(p=0.006)$.

\section{Conclusion}

LODDs $\geq-1.05$ was the only significant prognostic factor for both DFS and OS. In patients with LODDs $\geq-1.05$, intensified chemotherapy might be required, considering the high rate of DF.

\author{
Correspondence: Keun-Yong Eom, MD, PhD \\ Department of Radiation Oncology, \\ Seoul National University Bundang Hospital, \\ 82 Gumi-ro 173beon-gil, Bundang-gu, \\ Seongnam 13620, Korea \\ Tel: 82-31-787-7653 \\ Fax: 82-31-787-4019 \\ E-mail: 978sarang@hanmail.net \\ Received March 1, 2015 \\ Accepted June 16, 2015 \\ Published Online July 14, 2015
}

\begin{abstract}
Key words
Uterine cervical neoplasms, Lymph nodes,

Log odds of positive lymph node, Prognosis
\end{abstract}

\section{Introduction}

Introduction of adjuvant treatment has led to improved treatment outcomes in cervical cancer; however, $10 \%-20 \%$ of patients still experience treatment failure after radical surgery followed by recommended adjuvant treatment [1-3].
This recurrent disease has an unfavorable prognosis, with a 5 -year survival rate of $35 \%$ [4]. Given the grave prognosis, some researchers have evaluated the efficacy of consolidative chemotherapy (CTx) after adjuvant treatment in patients with high-risk factors such as lymph node (LN) status, parametrial (PM) invasion, bulky tumor, and resection margin $[5,6]$. However, the role of consolidative CTx remains unclear 
in these patients $[7,8]$. Identification of patients who might benefit from consolidative CTx after traditional adjuvant treatment first requires predictive markers for high risk of recurrence. However, unlike in the surgery-only treatment, there is no established prognostic factor for patients treated with radical hysterectomy followed by adjuvant treatment.

Underestimating the value of LN metastasis is a major weakness of the staging system for cervical cancer. The International Federation of Gynecology and Obstetrics (FIGO) staging system does not consider LN status, while the American Joint Committee on Cancer staging system simply stratifies it as N0 or N1 [9]. However, estimating risk for recurrence of other malignant solid tumors according to LN status suggests that patient's prognosis could be significantly influenced by LN burden, such as the number, location, or $\log$ odds of pathologic LNs. For example, Monk et al. [10] reported on the difference in clinical prognosis between single LN metastasis and $\geq 2$ LN metastasis after radical hysterectomy followed by adjuvant radiotherapy (RT). Kidd et al. [11], who studied LN staging by positron emission tomography, demonstrated that disease-free survival (DFS) could be stratified by the most distant level of LN metastasis as none, pelvic, para-aortic, or supraclavicular area $(\mathrm{p}<0.001)$. Demirci et al. [12] reported that the LN ratio (LNR) had clinical importance not only for overall survival (OS), but also for local control and DFS after adjuvant treatment in FIGO IB-II staged cervical cancer. More recently, the prognostic impacts of log odds of positive LNs (LODDs), the log of odds between number of positive LNs, and number of negative LNs have been studied in gastrointestinal cancers. However, the method for assessing LN status showing the most correlation with the prognosis for cervical cancer has not been determined. Therefore, we compared the prognostic value of various methods for assessing LN status, including simple pathologic $\mathrm{N}$ stage, the location and number of positive LNs, LNR, and LODDs in patients with high-risk cervical cancer treated with radical surgery and adjuvant treatment, in order to define subgroups at risk of high recurrence.

\section{Materials and Methods}

\section{Patients}

From a local database, consecutive 76 patients with histologically proven cervical cancer who had been referred for postoperative RT after radical surgery from January 2004 to June 2012 were identified. Ten patients who did not undergo radical hysterectomy and pelvic LN dissection were excluded. Among 66 patients, only 50 patients with one or more high risk factors, including tumor involvement at the surgical margin, PM invasion, or LN metastasis, were included in the analysis for homogeneity. Patients' medical records were reviewed for collection of data on demographics, clinicopathologic factors, treatment, and survival outcomes. Information collected included patients' complete blood count, including the differential and serum levels of tumor markers before surgery. The location and number of metastatic LNs were obtained from pathologic reports. According to the location of positive LNs, LNs were classified as upper LNs (common iliac LNs or higher) or lower LNs (below common iliac LNs). The LNR was calculated as the number of pathologic LNs divided by the number of harvested LNs. LODDs is defined as $\log ($ (No. of positive LNs +0.5$) /$ (No. of harvested LNs-No.of postivie LNs+0.5)).

All patients were restaged according to FIGO 2008 staging system [13]. Treatment-related complications were graded using the Common Terminology Criteria for Adverse Events ver. 4.03. This study was approved by our institutional review board.

\section{Treatment}

All patients underwent radical hysterectomy with pelvic LN dissection, with or without para-aortic LN sampling or dissection at the discretion of the treating physician. Postoperative RT was delivered with megavoltage photons by a linear accelerator, using a traditional four-field technique covering the whole pelvis. In six patients with microscopic metastasis in the paraaortic area, radiation portals for the paraaortic region were added. One patient with involvement of the vaginal resection margin underwent intracavitary RT to the vaginal stump with 15 Gy over five fractions. The median total dose and fraction were $50.4 \mathrm{~Gy}$ (range, 50 to 55.8 Gy) and 28 fractions (range, 25 to 31 fractions).

CTx was provided concurrently to 43 patients, and no patient underwent consolidative CTx after RT. Almost all patients (93\%, 40 of 43 patients) received platinum-based CTx with median four cycles (range, 2 to 6 cycles). Paclitaxel/ carboplatin was most commonly used (16 patients), and cisplatin as a single agent was administered weekly in 11 patients. The other regimens were 5-fluorouracil/ cisplatin (6 patients), gemcitabine/cisplatin (5 patients), docetaxel/ cyclophosphamide (3 patients), and paclitaxel/cisplatin (2 patients). Concurrent CTx was omitted in seven patients, because of poor performance or refusal.

\section{Clinical endpoint and statistical analysis}

The baseline for follow-up (BOF) was defined as the date of pathologic diagnosis of cervical cancer. DFS was the duration from $\mathrm{BOF}$ to the date of the most recent follow-up 
Table 1. Clinical characteristics

\begin{tabular}{|c|c|}
\hline Characteristic & No. $(\%)$ \\
\hline Age, median (range, yr) & $51(27$ to 78$)$ \\
\hline \multicolumn{2}{|l|}{ FIGO stage } \\
\hline $1 \mathrm{~A} 2$ & $2(4.0)$ \\
\hline 1B1 & $24(48.0)$ \\
\hline 1B2 & $10(20.0)$ \\
\hline 2A1 & $9(18.0)$ \\
\hline $2 \mathrm{~A} 2$ & $1(2.0)$ \\
\hline 2B & $4(8.0)$ \\
\hline \multicolumn{2}{|l|}{ Histology } \\
\hline Squamous cell carcinoma & $38(76.0)$ \\
\hline Adenocarcinoma & $7(14.0)$ \\
\hline Mucinous adenocarcinoma & $2(4.0)$ \\
\hline Adenosquamous carcinoma & $1(2.0)$ \\
\hline Glassy cell carcinoma & $1(2.0)$ \\
\hline Mucoepidermoid carcinoma & $1(2.0)$ \\
\hline \multicolumn{2}{|l|}{ Deep stromal invasion (outer $1 / 3$ ) } \\
\hline Negative & $6(12.0)$ \\
\hline Positive & $35(70.0)$ \\
\hline \multicolumn{2}{|l|}{ Parametrial extension } \\
\hline Negative & $20(40.0)$ \\
\hline Positive & $30(60.0)$ \\
\hline \multicolumn{2}{|l|}{ Bulky tumor (cm) } \\
\hline$\leq 4$ & $21(42.0)$ \\
\hline$>4$ & $29(58.0)$ \\
\hline \multicolumn{2}{|l|}{ Lymphovascular invasion } \\
\hline Negative & $11(22.0)$ \\
\hline Positive & $39(78.0)$ \\
\hline \multicolumn{2}{|l|}{ Perineural invasion } \\
\hline Negative & $43(86.0)$ \\
\hline Positive & $7(14.0)$ \\
\hline \multicolumn{2}{|l|}{ Pathologic N stage } \\
\hline No & $12(24.0)$ \\
\hline N1 & $38(76.0)$ \\
\hline \multicolumn{2}{|l|}{ Upper lymph node ${ }^{a)}$ involvement } \\
\hline Negative & $37(74.0)$ \\
\hline Positive & $13(26.0)$ \\
\hline \multicolumn{2}{|l|}{$\mathrm{LN}$ ratio $(\%)^{\mathrm{b})}$} \\
\hline$<10$ & $33(66.0)$ \\
\hline$\geq 10$ & $17(34.0)$ \\
\hline $\begin{array}{l}\text { Log odds of positive } \mathrm{LNs}^{\mathrm{c}} \text {, } \\
\text { median (range) }\end{array}$ & $-1.15(-2.09$ to -0.2$)$ \\
\hline$\geq-1.05$ & $22(44.0)$ \\
\hline$<-1.05$ & $28(56.0)$ \\
\hline Pretreatment NLR, median (range) & 2.09 (0.72 to 18.89$)$ \\
\hline$<2.1$ & $25(50.0)$ \\
\hline$\geq 2.1$ & $24(48.0)$ \\
\hline \multicolumn{2}{|l|}{ Adjuvant treatment } \\
\hline RT alone & $7(14.0)$ \\
\hline CCRT & $43(86.0)$ \\
\hline SCC, median $(\mathrm{U} / \mathrm{mL})$ & 2.60 \\
\hline
\end{tabular}

Table 1. Continued

\begin{tabular}{lc} 
Characteristic & No. $(\%)$ \\
CEA, median $(\mathrm{ng} / \mathrm{mL})$ & 1.07 \\
\hline
\end{tabular}

FIGO, International Federation of Gynecology and Obstetrics; LN, lymph node; NLR, neutrophil/lymphocyte ratio; $\mathrm{RT}$, radiotherapy; CCRT, concurrent chemoradiotherapy; SCC, squamous cell carcinoma antigen; CEA, carcinoembryonic antigen. ${ }^{\text {a)}}$ Upper lymph node: common iliac or para-aortic lymph node, ${ }^{b} \mathrm{LN}$ ratio: No. of pathologic LNs/No. of total LNs harvested, 'Log odds of positive LNs: $\log$ (No. of positive LNs/No. of negative LNs).

or the diagnosis date of recurrence. Recurrence was diagnosed through imaging studies; histologic confirmation was not mandatory. Recurrences were classified as locoregional failure (LRF) or distant failure (DF). LRF was defined as a failure in the intrapelvic and para-aortic areas, while DF was considered to be all relapses, except for LRF. OS was calculated from BOF to the date of the last follow-up or death. Survival data were collected through inquiries to the Resident Registration of the Ministry of Security and Public Administration of Republic of Korea.

Statistical analysis was performed using SPSS ver. 18 (SPSS Inc., Chicago, IL). DFS and OS curves were estimated using the Kaplan-Meier method, and the effects of each variable on survival were evaluated by log-rank tests. Multivariate analysis was performed using the Cox proportional hazard model with a backward conditional stepwise regression. The cut-off point for LODDs was selected based on the results of maximal chi-square method using $R$ ver. 3.03. $p$-values of less than 0.05 were considered statistically significant.

\section{Results}

Fifty patients with high-risk features were included and analyzed in this study. Median age of patients at diagnosis was 51 years (range, 27 to 78 years). Most patients ( $72 \%$ ) were in FIGO stage IA/B. The most common histology was squamous cell carcinoma $(76 \%)$. Other histologies included adenocarcinoma (7 patients), mucinous adenocarcinoma (2), adenosquamous carcinoma (1), glassy cell carcinoma (1), and mucoepidermoid carcinoma (1). Twelve patients showed no evidence of regional LN involvement. Other clinicopathologic factors are shown in Table 1.

The distribution of metastatic LNs in the 38 patients with positive LNs is shown in Table 2. The median number of pos- 
Table 2. Distribution of metastatic lymph nodes (LNs) in patients with $\mathrm{pN} 1$ ( $\mathrm{n}=38)$

\begin{tabular}{|llc} 
& No. (\%) & Distribution of pathologic LN \\
Pelvic node dissection & Total harvested node, median (range) & $30(10-79)$ \\
& Pathologic node, median (range) & $2(1-26)$ \\
& Obturator LN & $19(50.0)$ \\
& External iliac LN & $15(41.7)$ \\
& Internal iliac LN & $12(31.6)$ \\
Para-aortic LN examination $(\mathrm{n}=13)$ & Common iliac & $12(31.6)$ \\
& Parametrial LN & $3(7.9)$ \\
& Total harvested node, median (range) & $6(1-17)$ \\
\hline
\end{tabular}

Table 3. Treatment toxicity

\begin{tabular}{lccc} 
Toxicity & Grade 1/2 & Grade 3 & Grade 4 \\
Acute & & & \\
$\quad$ Gastrointestinal toxicity & $24(48)$ & - & - \\
$\quad$ Genitourinary toxicity & $10(20)$ & - & - \\
$\quad$ Neutropenia & $13(26)$ & $4(8)$ & $14(28)$ \\
Chronic & & & \\
$\quad$ Lymphedema & $18(36)$ & - & - \\
$\quad$ Peripheral neuropathy & $5(10)$ & - & - \\
$\quad$ Urinary incontinence & $2(4)$ & - & - \\
\hline
\end{tabular}

Values are presented as number (\%). Two patients underwent surgical intervention for adhesive ileus or vesicovaginal fistula.

itive LNs was two (range, 1 to 26), and involvement of obturator and external iliac LNs was most common. Thirteen patients $(19.7 \%)$ had metastatic upper LNs, 12 common iliac LNs, and six para-aortic LNs. Of these six patients, only one had skip metastasis to para-aortic LN without involvement of common iliac LNs. The median value of LODDs was -1.15 . Seventeen patients $(34 \%)$ had $\geq 10 \%$ LNRs, and $22(44 \%)$ $\geq-1.05$ of LODDs.

During the adjuvant treatment, the most common acute complication was gastrointestinal toxicity (48\%) followed by neutropenia $(36 \%)$ and genitourinary toxicity $(20 \%)$. These acute toxicities were resolved spontaneously within 3-4 months. Chronic lymphedema and peripheral neuropathy were reported in $36 \%$ and $10 \%$, respectively. Two patients suffered from sustained urinary incontinence (Table 3).

\section{Treatment failure}

The median follow-up duration was 80 months (range, 6 to 123 months). The estimated 5-year LRF-free survival rate was $89.5 \%$, while the distant metastasis-free survival rate was $78.5 \%$. Eleven patients (22\%) experienced treatment failure. DF was dominant, occurring in 10 cases. Six cases were DF only, four were DF combined with LRF, and one was isolated LRF. The lungs were the most common site of DF (4 of 10 patients). Four simultaneous LRFs occurred in the RT field: the common iliac LN, paraaortic LN, bladder wall, and vaginal stump. These LRFs received at least $50.4 \mathrm{~Gy}$, and up to 55.8 Gy were delivered to the para-aortic failure site by conventional fractionation. Isolated LRFs, which were not in the RT field, occurred in the lower vagina, adjacent to the vulva.

Among the 11 patients with treatment failure, five died a median of 10.6 months after failure. Two patients were still alive and receiving salvage CTx at the most recent followup, and the others (isolated DF, 2 patients; isolated LRF, 1 patient) were successfully salvaged and had maintained a state of no evidence of disease.

\section{Analysis of DFS and OS}

The 5-year DFS and OS rates were $76.1 \%$ and $86.4 \%$, respectively. In univariate analysis for DFS (Table 4 ), perineural invasion $(p<0.012), \geq 2$ LN metastasis $(p=0.030)$, LNR $\geq 10 \%(p=0.003)$, upper LN metastasis $(p=0.002)$, and $\geq-1.05$ LODDs $(p<0.001)$ (Fig. 1A) were statistically significant unfavorable factors for DFS. Among the methods used for assessment of LN status, LODDs was the most powerful indicator associated with DFS. Lymphovascular invasion (LVI) and pN1 showed a trend toward poor DFS (LVI, $\mathrm{p}=0.053 ; \mathrm{pN1}, \mathrm{p}=0.059)$. Regarding OS, LODDs again was the only statistically significant prognostic factor $(p=0.006)$ (Fig. 1B). Neither preoperative tumor markers nor the preoperative neutrophil/lymphocyte ratio was a prognostic parameter for DFS or OS.

Subgroup analyses were performed to determine whether 
Table 4. Univariate analysis for disease-free survival (DFS) and overall survival (OS)

\begin{tabular}{|c|c|c|c|c|}
\hline Variable & 5-yr DFS (\%) & p-value ${ }^{a)}$ & $5-\mathrm{yr}$ OS $(\%)$ & $p$-value ${ }^{a}$ \\
\hline \multicolumn{5}{|l|}{ Age $(y r)$} \\
\hline$\leq 50$ & 78.0 & 0.831 & 91.4 & 0.460 \\
\hline$>50$ & 74.1 & & 82.5 & \\
\hline \multicolumn{5}{|l|}{ Histology } \\
\hline Squamous cell carcinoma & 74.3 & 0.718 & 85.0 & 0.724 \\
\hline Others & 83.3 & & 91.7 & \\
\hline \multicolumn{5}{|l|}{ Deep stromal invasion } \\
\hline Negative & 100 & 0.149 & 100 & 0.311 \\
\hline Positive & 69.0 & & 83.4 & \\
\hline \multicolumn{5}{|l|}{ Bulky tumor $(\mathrm{cm})$} \\
\hline$\leq 4$ & 78.6 & 0.686 & 82.4 & 0.700 \\
\hline$>4$ & 74.5 & & 88.7 & \\
\hline \multicolumn{5}{|l|}{ Lymphovascular invasion } \\
\hline Negative & 100 & 0.053 & 100 & 0.157 \\
\hline Positive & 69.1 & & 82.2 & \\
\hline \multicolumn{5}{|l|}{ Parametrial invasion } \\
\hline Negative & 89.1 & 0.084 & 95.0 & 0.195 \\
\hline Positive & 67.3 & & 79.8 & \\
\hline \multicolumn{5}{|l|}{ Perineural invasion } \\
\hline Negative & 82.2 & 0.012 & 87.0 & 0.801 \\
\hline Positive & 38.1 & & 83.3 & \\
\hline \multicolumn{5}{|l|}{ Pathologic N stage } \\
\hline No & 100 & 0.059 & 100 & 0.197 \\
\hline N1 & 69.6 & & 83.0 & \\
\hline \multicolumn{5}{|l|}{ No. of LN invasion } \\
\hline$<2$ & 90.0 & 0.030 & 87.0 & 0.801 \\
\hline$\geq 2$ & 59.6 & & 83.3 & \\
\hline \multicolumn{5}{|l|}{ Upper $\mathbf{L N}^{\mathrm{b})}$ involvement } \\
\hline Negative & 88.2 & 0.002 & 90.8 & 0.197 \\
\hline Positive & 46.2 & & 75.0 & \\
\hline \multicolumn{5}{|l|}{ LN ratio $(\%)^{\mathrm{c}}$} \\
\hline$<10$ & 89.7 & 0.003 & 92.8 & 0.092 \\
\hline$\geq 10$ & 52.3 & & 75.3 & \\
\hline \multicolumn{5}{|l|}{ Log odds of positive LNs ${ }^{d)}$} \\
\hline$\geq-1.05$ & 54.2 & $<0.001$ & 71.5 & 0.006 \\
\hline$<-1.05$ & 96.4 & & 100 & \\
\hline \multicolumn{5}{|l|}{ Pretreatment NLR } \\
\hline$<2.1$ & 74.0 & 0.646 & 85.8 & 0.875 \\
\hline$\geq 2.1$ & 83.3 & & 86.8 & \\
\hline \multicolumn{5}{|l|}{ SCC } \\
\hline$\leq 2$ & 87.8 & 0.145 & 93.8 & 0.256 \\
\hline$>2$ & 72.1 & & 82.3 & \\
\hline
\end{tabular}

LN, lymph node; NLR, neutrophil/lymphocyte ratio; SCC, squamous cell carcinoma antigen. ${ }^{a}$ p-value by log-rank test, b) Upper LN included common iliac or para-aortic lymph node, "'LN ratio: No. of pathologic LNs / No. of total LNs harvested, d)Log odds of positive LNs: $\log$ (No. of positive LNs / No. of negative LNs).

survival differences associated with LN status (number of positive LNs, LNR, upper LN metastasis, and LODDs) remained after separating patients with pN0 (Fig. 2). The
DFS curves of patients with single LN metastasis, LNR $<10 \%$, and only lower pelvic LN metastasis did not differ from those of patients with $\mathrm{pN} 0$. The DFS curve of patients 
A

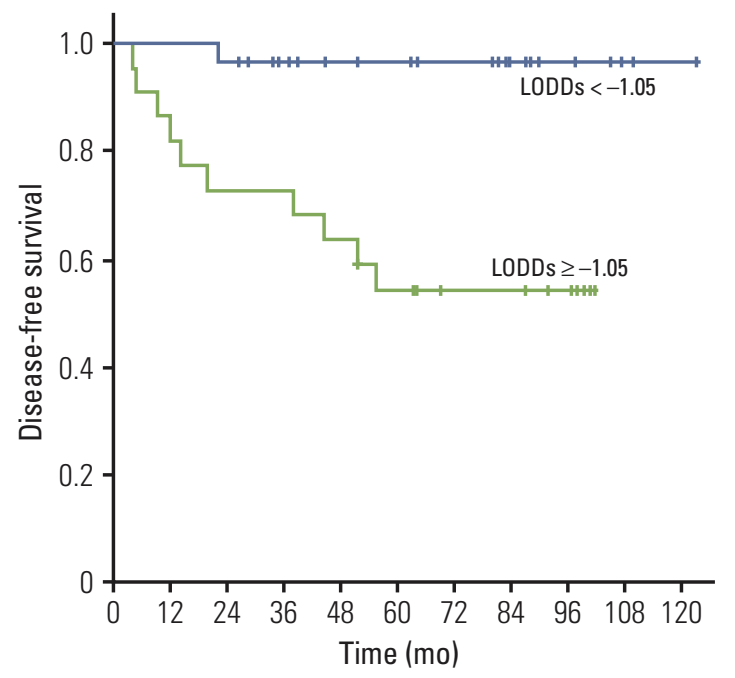

B

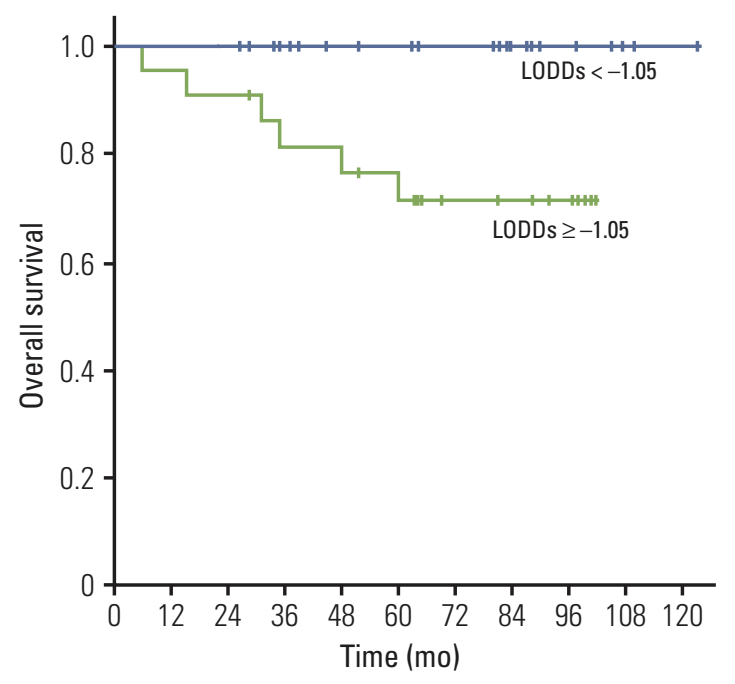

Fig. 1. Disease-free survival (A) and overall survival (B) according to log odds of positive lymph nodes (LODDs).

with LODDs $<-1.05$ was almost the same as that of $\mathrm{pN} 0$ (Fig. 2A). Patients with LODDs $\geq-1.05$ (5-year, 93.8\% vs. 54.2\%; $\mathrm{p}=0.015$ ) (Fig. 2A), upper LN metastasis (5-year, $83.1 \%$ vs. $46.2 \%$; $\mathrm{p}=0.023$ ) (Fig. 2B), and LNR $\geq 10 \%$ (5-year, $84.4 \%$ vs. $52.3 \%$; $\mathrm{p}=0.036$ ) (Fig. 2C) had significantly shorter DFS than those without these factors. Patients with $\geq 2$ LN metastasis also had shorter DFS than those with single LN metastasis, but the difference was not statistically significant (5-year, 83.3\% vs. 59.6\%; p=0.204) (Fig. 2D).

Variables with statistical significance in univariate analysis for DFS were included in multivariate analysis. In multivariate analysis, DFS was significantly affected by $\geq-1.05$ LODDs ( $p=0.011$; hazard ratio, 0.069 ; $95 \%$ confidence interval, 0.01 to 0.54 ), which was the only significant prognostic factor for DFS. The prognostic significance of location, $\geq 2$ LNs metastasis, and LNR disappeared after adjusting for other factors.

\section{Discussion}

Our data show that patients with LODDs $\geq-1.05$ had significant risk for disease recurrence, specifically DF. Among the various methods used for assessment of LN state, LODDs was determined to be the strongest predictor of both recurrence and survival. However, other parameters related to LN status also seem to be important prognostic factors in patients with high risk cervical cancer as well as LODDs. The traditional prognostic factors suggested by surgical series, including bulky tumors, deep stromal invasion, resection margin involvement, $\mathrm{PM}$ invasion, and $\mathrm{pN}$ stage, did not predict the treatment outcomes in the current study. Their clinical impact might be attenuated by the adjuvant treatment.

In most solid tumors, $\mathrm{N}$ stages are categorized according to the number or region of pathologic LNs, which are well correlated with prognosis in general. In this context, many researchers have attempted to determine the clinical significance of nodal involvement of cervical cancer. For example, Monk et al. [10] demonstrated that patients with two or more positive LNs had a significantly lower survival rate after radical surgery followed by only postoperative RT (5-year OS, $55 \%$ vs. $79 \%$; $\mathrm{p}=0.01$ ). This unfavorable outcome was offset by the addition of CTx (5-year OS, 75\% vs. 83\%; $\mathrm{p}=0.37$ ). Tsai et al. [5], who also reported the negative impact of two or more LNs on survival after adjuvant RT, and pointed to the significance of the location of positive LNs, categorized LNs as lower and upper LNs and showed that metastasis to upper LN was associated with a higher incidence of distant metastasis ( $50 \%$ vs. $16 \%$, $\mathrm{p}=0.03$ ). In similar results, Takeda et al. [14] reported that common iliac LNs significantly affected survival (5-year OS, approximately $40 \%$ vs. $80 \%$; $\mathrm{p}=0.003$ ) after adjuvant RT. When considering the hierarchical tendency of lymphatic spread in cervical cancer, the involvement of upper pelvic LN may worsen patients' treatment outcomes. However, in the current study, despite their significance in univariate analysis, the importance of metastasis to $\geq 2$ LNs and upper LN disappeared in multivariate 
A

B
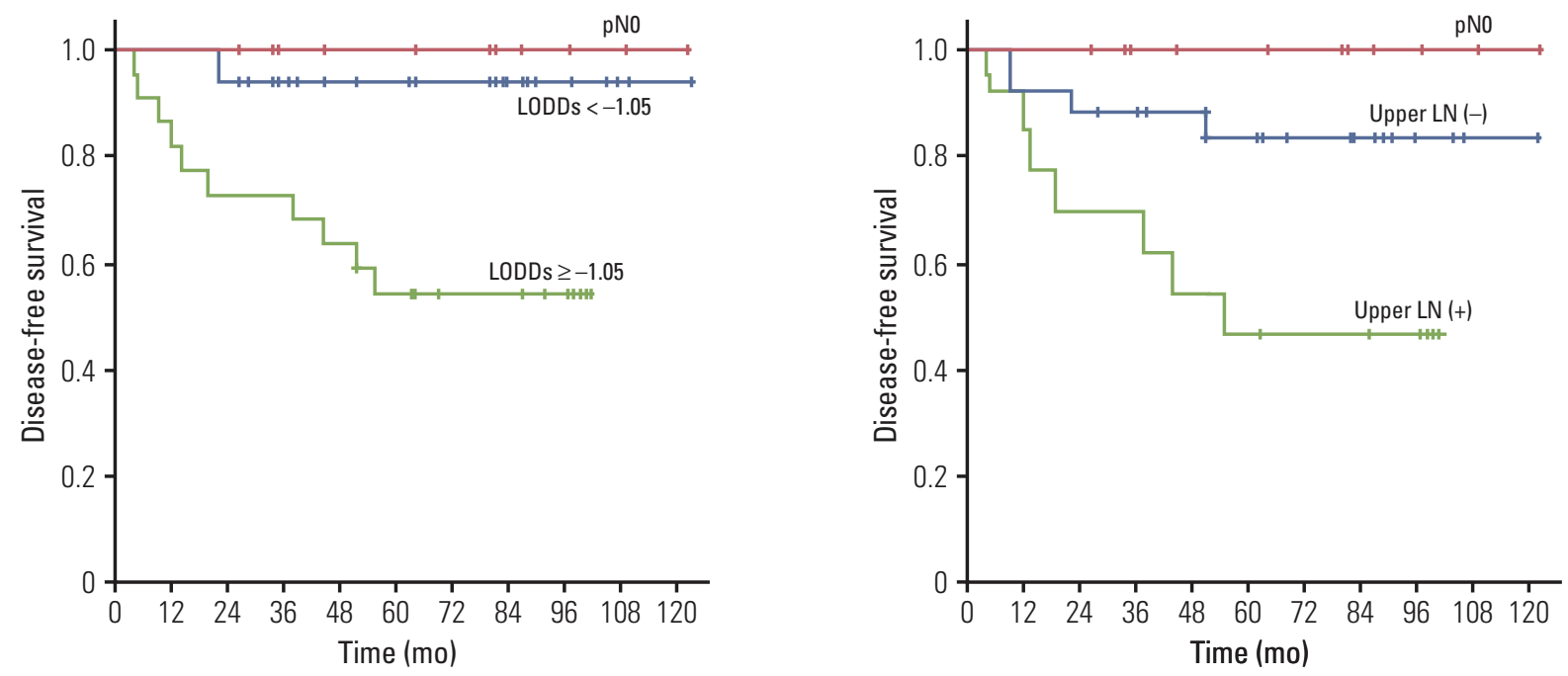

C
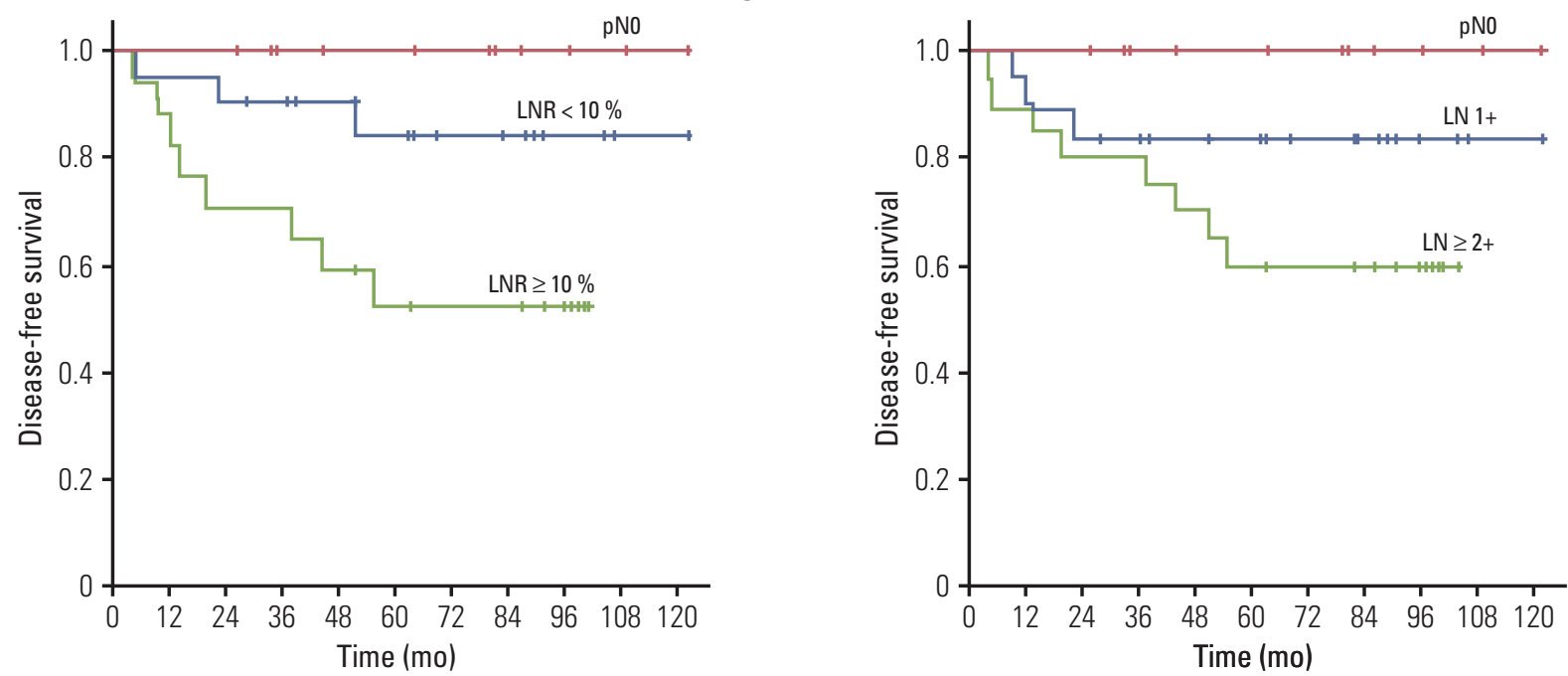

Fig. 2. Disease-free survival according to log odds of positive lymph nodes (LNs) (LODDs) (A), upper LN metastasis (B), lymph node ratio (LNR) (C), and number of positive LNs (D).

analysis. This change might be due to the close correlation between $\geq 2$ LNs or upper LN metastasis and LODDs $\geq-1.05$ (Fisher exact test, $\mathrm{p}<0.001$ ).

Polterauer et al. [15] suggested LN density as an independent prognostic factor for DFS $(\mathrm{p}=0.01)$ and OS $(\mathrm{p}=0.05)$. Their definition of LN density was the same as the LNR in the current study, while the cut-off value was 10\% based on a study of esophageal cancer. Our results of univariate analysis also showed a difference in DFS according to LNR. However, this prognostic significance was not observed in multivariate analysis, perhaps because of the close correlation of LNR to
LODDs. All patients in the LNR $\geq 10 \%$ group were also included in the LODDs $\geq-1.05$ group (Fisher exact test, $\mathrm{p}<0.001$ ). Both the study by Polterauer et al. [15] and the current study were retrospective studies, which included a small number of patients. Therefore, a large prospective study is needed to address the significance of the LNR.

LODDs is not a familiar prognostic factor in cervical cancer. To the best of the authors' knowledge, the prognostic value of LODDs has not been tested in cervical cancer. The efficacy of LODDs in gastric and colorectal cancer was already reported in large scale studies and Wang et al. [16] 
demonstrated the prognostic superiority of LODDs to LNR. LODDs is a parameter requiring complex calculation and is less intuitive than LNR. However, LODDs' strength lies in their ability to discriminate patients with equal LNRs. In particular, Sun et al. [17] demonstrated that LODDs was still heterogeneous even though LNR was equal as 0 or 1 . This finding suggested the potential of LODDs to distinguish different prognosis among patients with the same $\mathrm{N}$ stage.

In the current study, among the methods used for assessment of LN status, LODDs was the most powerful indicator associated with DFS. LODDs identified the subgroup that had a prognosis similar to that of patients with $\mathrm{pN} 0$, despite the presence of metastatic LNs (Fig. 2A). Patients with LODDs $\geq-1.05$ also had decreased OS. In addition, among 22 patients with LODDs $\geq-1.05$, nine $(40.9 \%)$ experienced DF. Similarly, $90 \%$ of patients with DF had a value of LODDs $\geq-1.05$. These findings suggest that patients with LODDs $\geq-1.05$ might have a high risk of DF and, therefore, should be considered for further systemic CTx.

In the current study, the majority of treatment failures were DF, consistent with the findings of previous studies reported in the literature $[18,19]$. No relationship was observed between the RT field and the dose and recurrence site, and the incidence of LRF was acceptably low. These findings suggest that postoperative RT is delivered adequately. Further improvement of the outcomes might require strengthening CTx, instead of RT. Identification of patients at risk is important even after adjuvant RT or chemoradiotherapy. Kim et al. [20] proposed a nomogram for predicting the risk of recurrence in early-stage patients (I-IIA) after radical surgery with or without adjuvant treatment. This model is based on stage, number of pathologic LNs, PM invasion, and depth of invasion. Using similar variables, the Korean Radiation Oncology Group Study 13-03 more recently suggested a nomogram for prediction of OS after postoperative treatment [21]. However, these models have employed wellknown traditional factors identified only through surgical series. The newly emerging prognostic factors after completion of adjuvant treatment also need to be evaluated.
The current study has several limitations. First, many inherent biases stem from its nature as a retrospective study. Second, the number of patients studied might be not large enough to support definite conclusions. There is a probability that two or more LNs metastasis, upper LN involvement, or LNR $\geq 10 \%$ also has clinical significance in consideration of statistical correlation of these parameters with LODDs. Although LODDs was the most meaningful factor in the result of multivariate analysis, it should be validated through study comprising more patients. Finally, the study population contained adjuvant RT only groups in spite of high risk features, which caused treatment heterogeneity. However, conversely, this study population is more realistic than CCRT alone in this clinical setting. The literature has dealt little with LODDs as an important prognostic factor, thus this study is the first to demonstrate the prognostic value of LODDs in patients with cervical cancer.

\section{Conclusion}

In conclusion, LODDs $\geq-1.05$ is an independent prognostic factor for DFS in high-risk patients who underwent radical surgery followed by adjuvant treatment. OS also showed a significant decrease in patients with LODDs $\geq-1.05$. Among the various methods used for assessment of LN status, LODDs was the most powerful predictor associated with both recurrence and OS. Therefore, considering the high rate of DF, intensified CTx might be required in patients with LODDs $\geq-1.05$.

\section{Conflicts of Interest}

Conflict of interest relevant to this article was not reported.

\section{References}

1. Rotman M, Sedlis A, Piedmonte MR, Bundy B, Lentz SS, Muderspach LI, et al. A phase III randomized trial of postoperative pelvic irradiation in Stage IB cervical carcinoma with poor prognostic features: follow-up of a gynecologic oncology group study. Int J Radiat Oncol Biol Phys. 2006;65:169-76.

2. Song S, Song C, Kim HJ, Wu HG, Kim JH, Park NH, et al. 20 year experience of postoperative radiotherapy in IB-IIA cervical cancer patients with intermediate risk factors: impact of treatment period and concurrent chemotherapy. Gynecol Oncol. 2012;124:63-7.

3. Kim WY, Chang SJ, Chang KH, Yoo SC, Chun M, Ryu HS. Differing prognosis of cervical cancer patients with high risk of treatment failure after radical hysterectomy warrants trial treatment modification. J Gynecol Oncol. 2009;20:17-21.

4. Piura B, Rabinovich A, Friger M. Recurrent cervical carcinoma after radical hysterectomy and pelvic lymph node dissection: 
a study of 32 cases. Eur J Gynaecol Oncol. 2008;29:31-6.

5. Tsai CS, Lai CH, Wang CC, Chang JT, Chang TC, Tseng CJ, et al. The prognostic factors for patients with early cervical cancer treated by radical hysterectomy and postoperative radiotherapy. Gynecol Oncol. 1999;75:328-33.

6. Lin HH, Cheng WF, Chan KW, Chang DY, Chen CK, Huang SC. Risk factors for recurrence in patients with stage IB, IIA, and IIB cervical carcinoma after radical hysterectomy and postoperative pelvic irradiation. Obstet Gynecol. 1996;88: 274-9.

7. Kim HS, Kim MK, Kim HJ, Han SS, Kim JW. Phase II study of consolidation chemotherapy after adjuvant or primary concurrent chemoradiation using paclitaxel and carboplatin to treat high-risk early-stage or locally advanced cervical cancer. Cancer Res Treat. 2012;44:97-103.

8. Lee JW, Kim BG, Lee SJ, Lee SH, Park CS, Lee JH, et al. Preliminary results of consolidation chemotherapy following concurrent chemoradiation after radical surgery in high-risk early-stage carcinoma of the uterine cervix. Clin Oncol (R Coll Radiol). 2005;17:412-7.

9. Compton CC, Byrd DR, Garcia-Aguilar J, Kurtzman SH, Olawaiye A, Washington MK. AJCC cancer staging atlas: a companion to the seventh editions of the AJCC cancer staging manual and handbook. New York: Springer; 2012.

10. Monk BJ, Wang J, Im S, Stock RJ, Peters WA 3rd, Liu PY, et al. Rethinking the use of radiation and chemotherapy after radical hysterectomy: a clinical-pathologic analysis of a Gynecologic Oncology Group/Southwest Oncology Group/Radiation Therapy Oncology Group trial. Gynecol Oncol. 2005; 96:721-8.

11. Kidd EA, Siegel BA, Dehdashti F, Rader JS, Mutch DG, Powell MA, et al. Lymph node staging by positron emission tomography in cervical cancer: relationship to prognosis. J Clin Oncol. 2010;28:2108-13.

12. Demirci S, Ozsaran Z, Ozsaran A, Yavas F, Demircioglu B, Hanhan $M$, et al. Evaluation of treatment results and prognostic factors in early-stage cervical carcinoma patients treated with postoperative radiotherapy or radiochemotherapy. Eur
J Gynaecol Oncol. 2012;33:62-7.

13. Pecorelli S, Zigliani L, Odicino F. Revised FIGO staging for carcinoma of the cervix. Int J Gynaecol Obstet. 2009;105:107-8.

14. Takeda N, Sakuragi N, Takeda M, Okamoto K, Kuwabara M, Negishi $\mathrm{H}$, et al. Multivariate analysis of histopathologic prognostic factors for invasive cervical cancer treated with radical hysterectomy and systematic retroperitoneal lymphadenectomy. Acta Obstet Gynecol Scand. 2002;81:1144-51.

15. Polterauer S, Hefler L, Seebacher V, Rahhal J, Tempfer C, Horvat $R$, et al. The impact of lymph node density on survival of cervical cancer patients. Br J Cancer. 2010;103:613-6.

16. Wang J, Hassett JM, Dayton MT, Kulaylat MN. The prognostic superiority of log odds of positive lymph nodes in stage III colon cancer. J Gastrointest Surg. 2008;12:1790-6.

17. Sun Z, Xu Y, Li DM, Wang ZN, Zhu GL, Huang BJ, et al. Log odds of positive lymph nodes: a novel prognostic indicator superior to the number-based and the ratio-based $\mathrm{N}$ category for gastric cancer patients with R0 resection. Cancer. 2010;116: 2571-80.

18. Peters WA 3rd, Liu PY, Barrett RJ 2nd, Stock RJ, Monk BJ, Berek JS, et al. Concurrent chemotherapy and pelvic radiation therapy compared with pelvic radiation therapy alone as adjuvant therapy after radical surgery in high-risk early-stage cancer of the cervix. J Clin Oncol. 2000;18:1606-13.

19. Soisson AP, Soper JT, Clarke-Pearson DL, Berchuck A, Montana G, Creasman WT. Adjuvant radiotherapy following radical hysterectomy for patients with stage IB and IIA cervical cancer. Gynecol Oncol. 1990;37:390-5.

20. Kim MK, Jo H, Kong HJ, Kim HC, Kim JW, Kim YM, et al. Postoperative nomogram predicting risk of recurrence after radical hysterectomy for early-stage cervical cancer. Int J Gynecol Cancer. 2010;20:1581-6.

21. Lee HJ, Han S, Kim YS, Nam JH, Kim HJ, Kim JW, et al. Individualized prediction of overall survival after postoperative radiation therapy in patients with early-stage cervical cancer: a Korean Radiation Oncology Group study (KROG 13-03). Int J Radiat Oncol Biol Phys. 2013;87:659-64. 